\title{
COMMENTARIES
}

\section{Biowaiver Monographs for Immediate Release Solid Oral Dosage Forms: Aciclovir}

\author{
J. ARNAL, ${ }^{1}$ I. GONZALEZ-ALVAREZ, ${ }^{1}$ M. BERMEJO, ${ }^{1}$ G.L. AMIDON ${ }^{2}$ H.E. JUNGINGER, ${ }^{3}$ S. KOPP, ${ }^{4 \dagger}$ \\ K.K. MIDHA, ${ }^{5}$ V.P. SHAH, ${ }^{6}$ S. STAVCHANSKY, ${ }^{7}$ J.B. DRESSMAN, ${ }^{8}$ D.M. BARENDS ${ }^{9 \dagger}$ \\ ${ }^{1}$ Facultad de Farmacia, Universidad de Valencia, Burjassot 46100, Valencia, Spain \\ ${ }^{2}$ College of Pharmacy, University of Michigan, Ann Arbor, Michigan \\ ${ }^{3}$ Faculty of Pharmaceutical Sciences, Naresuan University, Phitsanulok, Thailand \\ ${ }^{4}$ World Health Organization, Geneva, Switzerland \\ ${ }^{5}$ University of Saskatchewan, Saskatoon, Saskatchewan, Canada \\ ${ }^{6}$ International Pharmaceutical Federation FIP, Den Haag, The Netherlands \\ ${ }^{7}$ Pharmaceutical Division, College of Pharmacy, University of Texas at Austin, Austin, Texas \\ ${ }^{8}$ Institute of Pharmaceutical Technology, J.W. Goethe University, Frankfurt am Main, Germany \\ ${ }^{9}$ RIVM, National Institute for Public Health and the Environment, Bilthoven, The Netherlands
}

Received 30 January 2008; accepted 25 February 2008

Published online 18 April 2008 in Wiley InterScience (www.interscience.wiley.com). DOI 10.1002/jps.21392

\begin{abstract}
Literature data relevant to the decision to allow a waiver of in vivo bioequivalence (BE) testing (biowaiver) for the approval of immediate release (IR) solid oral dosage forms containing aciclovir are reviewed. Aciclovir therapeutic use and therapeutic index, pharmacokinetic properties, data related to the possibility of excipient interactions and reported BE/bioavailability (BA) studies were also taken into consideration in order to ascertain whether a biowaiver can be recommended. According to the Biopharmaceutics Classification System (BCS) and considering tablet strengths up to $400 \mathrm{mg}$, aciclovir would be BCS Class III. However, in some countries also $800 \mathrm{mg}$ tablets are available which fall just within BCS Class IV. Aciclovir seems not to be critical with respect to a risk for bioinequivalence, as no examples of bioinequivalence have been identified. It has a wide therapeutic index and is not used for critical indications. Hence, if: (a) the test product contains only excipients present in aciclovir solid oral IR drug products approved in ICH or associated countries, for instance as presented in this article; and (b) the comparator and the test product both are very rapidly dissolving, a biowaiver for IR aciclovir solid oral drug products is considered justified for all tablet strengths. (c) 2008 Wiley-Liss, Inc. and the American Pharmacists Association J Pharm Sci 97:5061-5073, 2008
\end{abstract}

Keywords: absorption; aciclovir; bioequivalence; biopharmaceutics classification system (BCS); permeability; solubility; regulatory science

${ }^{\dagger}$ This article reflects the scientific opinion of the authors and not the policies of regulating agencies.

Correspondence to: D.M. Barends (Telephone: $+31-30-$ 2744209; Fax: +31-30-2744462; E-mail: dirk.barends@rivm.nl)
Journal of Pharmaceutical Sciences, Vol. 97, 5061-5073 (2008)

(c) 2008 Wiley-Liss, Inc. and the American Pharmacists Association 


\section{INTRODUCTION}

A biowaiver monograph of aciclovir based on literature data is presented. The risks of basing a $\mathrm{BE}$ assessment on in vitro rather than in vivo study results for the approval of new IR solid oral dosage forms containing aciclovir ("biowaiving"), including both reformulated products and new multisource products, are evaluated under consideration of its biopharmaceutical and clinical properties. This evaluation refers to drug products containing aciclovir as the only active pharmaceutical ingredient (API) and not to combination drug products.

The purpose and scope of this series of monographs have been previously discussed. ${ }^{1}$ Summarized in few words, the aim is to evaluate all pertinent data available from literature sources for a given API to assess the risks associated with a biowaiver. For these purposes, risk is defined as the probability of an incorrect biowaiver decision as well as the consequences of the decision in terms of public health and individual patient risks. On the basis of these considerations, a recommendation can be made as to whether a biowaiver is advisable or not. This systematic approach to recommend or advise against a biowaiver decisions is referred to in the recently published World Health Organization (WHO) Guideline. ${ }^{2}$ It is to be understood that these monographs do not simply apply the WHO, ${ }^{2}$ FDA, ${ }^{3}$ and EMEA Guidances, ${ }^{4}$ but also aim to serve as a critical validation of these regulatory documents. Biowaiver monographs have already been published for acetaminophen (INN: paracetamol),${ }^{5}$ acetazolamide, ${ }^{6}$ amitriptyline,${ }^{7}$ atenolol, ${ }^{1}$ chloroquine,${ }^{8}$ cimetidine,${ }^{9}$ ethambutol, ${ }^{10}$ ibuprofen, ${ }^{11}$ isoniazid, ${ }^{12}$ prednisolone,${ }^{13}$ prednisone,${ }^{14}$ pyranisamide, ${ }^{15}$ propranolol, ${ }^{1}$ ranitidine, ${ }^{16}$ and verapamil. ${ }^{1}$ They are also available on-line at www.fip.org/bcs.

\section{EXPERIMENTAL}

Literature data was assessed from PubMed ${ }^{17}$ and Micromedex ${ }^{18}$ databases. Keywords used for searching, in various combinations were: aciclovir, acyclovir, solubility, permeability, dissolution. Information was also obtained from regulatory documents published by the EMEA, ${ }^{4}$ the FDA, ${ }^{3}$ and the WHO. ${ }^{2,19}$ The $\mathrm{USP}^{20}$ and the European Pharmacopoeia ${ }^{21}$ were also consulted when necessary.

\section{GENERAL CHARACTERISTICS}

\section{Name}

The INN and WHO chemical name for aciclovir is 2-amino-1,9-dihydro-9-[(2-hydroxyethoxy)methyl]$6 \mathrm{H}$-purin-6-one, or 9-[(2-hydroxyethoxy)methyl]guanine. $^{20,22}$ Other names are: acyclovir, acycloguanosine and ACV. ${ }^{23}$ Its molecular formula is $\mathrm{C}_{8} \mathrm{H}_{11} \mathrm{~N}_{5} \mathrm{O}_{3}$, and its molecular weight is 225.21 $\mathrm{g} / \mathrm{mol}$. Its CAS number is 59277-89-3. ${ }^{20,22}$<smiles>Nc1nc(=O)c2ncn(COCCO)c2[nH]1</smiles>

\section{Therapeutic Indications, Therapeutic Index and Toxicity}

Aciclovir is used orally for the treatment and prophylaxis of initial and recurrent episodes of genital and labial herpes and for the acute treatment of herpes zoster for the treatment of varicella (chickenpox) in immunocompetent individuals. ${ }^{24,25}$ Its defined daily dose, either orally or parenterally, is $4 \mathrm{~g} .{ }^{26}$ Oral administration up to doses of $4800 \mathrm{mg} /$ day is usually well tolerated although high-dose treatment with oral aciclovir for herpes zoster results can cause more side effects than low-dose treatments. ${ }^{27}$ Several patients have ingested up to 100 capsules, corresponding to $20 \mathrm{~g}$ of aciclovir, with no apparent adverse effects, ${ }^{28}$ probably due to the limited solubility and absorption characteristics. Neurotoxicity may be seen with high doses in patients with compromised renal function. ${ }^{28-30}$ Neurotoxicity can include coma, confusion, 
delirium, encephalopathy, hallucinations, paresthesias, psychosis, seizures, or tremor. Neurologic adverse reactions usually occur within 1-2 days of achieving the maximum aciclovir concentration and may not be directly correlated with the aciclovir serum concentrations at the time the toxic effects appear. The threshold for neurotoxicity was reported to be $4.5 \mu \mathrm{g} / \mathrm{mL}$, whereas the normal peak range is $0.4-2 \mu \mathrm{g} / \mathrm{mL} .{ }^{28}$ Diarrhea, nausea and vomiting, and elevated serum creatinine levels may be observed in conjunction with plasma levels over $20 \mathrm{mcg} / \mathrm{mL}$, but recede when the dose is reduced. ${ }^{28}$

Serious neurological and/or psychiatric adverse effects been reported in a few patients administered bolus injections of high doses intravenous aciclovir. ${ }^{27,28,30}$ Aciclovir has low solubility in urine and aciclovir crystals may precipitate in the renal tubules if the solubility is exceeded in the intratubular fluid, resulting in renal dysfunction, renal failure or anuria, ${ }^{24,28}$ but this crystalluria is most likely to occur only during administration of large, parenteral doses. All these latter adverse drug reactions are not associated with the oral administration and hence are not relevant for this monograph.

\section{PHYSCOCHEMICAL PROPERTIES}

\section{Salts, Esters, Polymorphs, Hydrates}

Aciclovir is commonly used as the free acid form in solid oral dosage forms, whereas the sodium salt is used in parenteral dosage forms. ${ }^{22,23}$ Valaciclovir, the L-valyl ester of aciclovir, has been used orally to increase its BA. ${ }^{31-36}$ Several dipeptide ester prodrugs are being tested to assess their usefulness in therapeutics, ${ }^{37,38}$ as well as some bile acid conjugate prodrugs ${ }^{39}$ and a phospholipid prodrug. ${ }^{40}$ The scope of this monograph is restricted to drug products containing aciclovir as the free acid.

Aciclovir is normally present in a hydrated form consisting of three aciclovir molecules to two molecules of water ${ }^{41}$ corresponding to a theoretical water content of about 5\%, but dose and solubility are normally expressed in units of anhydrous aciclovir. ${ }^{20,42} \mathrm{~A}$ stable anhydrous form can be obtained by drying hydrated aciclovir at temperatures above $150^{\circ} \mathrm{C} .{ }^{41}$ Although only slight and insignificant differences in solubility values exist between these two forms, the anhydrous form of aciclovir possesses poorer dissolution properties than the hydrated form. ${ }^{41}$

\section{Solubility}

Aciclovir is described as "slightly soluble in water" in different Pharmacopoeias. ${ }^{20,21}$ Most data found in literature are calculated at room temperature $\left(22-25^{\circ} \mathrm{C}\right)$, reporting solubility values that range from 1.2 to $1.6 \mathrm{mg} / \mathrm{mL}^{41,43-47} \mathrm{In}$ water at $37^{\circ} \mathrm{C}$, solubility has been reported to be $2.5 \mathrm{mg} / \mathrm{mL} .{ }^{24}$ A plot of the $\mathrm{pH}$-solubility in de-ionized water at $37^{\circ} \mathrm{C}$ was published, showing the solubility to vary slightly with $\mathrm{pH}$, with a lowest solubility of $2.3 \mathrm{mg} /$ $\mathrm{mL}$ at $\mathrm{pH} 5.8$ at $37^{\circ} \mathrm{C}{ }^{48}$ The solubility data in Table 1 were obtained from this $\mathrm{pH}$-solubility profile.

\section{Partition Coefficient}

The partition coefficient $(\log P)$ in $n$-octanol at $22^{\circ} \mathrm{C}$ is $-1.57 .45,49$

At $25^{\circ} \mathrm{C}, \log P$ and $\log D$ were both reported to be $-1.8^{44,50}$ at $\mathrm{pH}$ 6.8. Calculations by Kasim et al. using fragmentation methods based on atomic contributions to lipophilicity and by using the $C \log P^{\circledR}$ program (version 3.0, Biobyte Corp., Claremont, CA, http://www.biobyte.com) gave values of -2.42 for $C \log P^{\circledR}$, and -1.59 for $\log P$, respectively. ${ }^{51}$

\section{$\mathrm{p} K_{\mathrm{a}}$}

Aciclovir is an ampholyte with both weak acid and basic groups. Common literature $\mathrm{p} K_{\mathrm{a}}$ values for aciclovir are 2.27 and 9.25 , but it is not stated at which temperature they were measured. ${ }^{23,24}$ Balon et al. ${ }^{50}$ reported $\mathrm{p} K_{\mathrm{a}}$ values of 2.16 and 9.04 at $37^{\circ} \mathrm{C}$.

Table 1. Solubility Data at $37^{\circ} \mathrm{C}$ and the Corresponding Dose/Solubility $(D / S)$ Ratios for Different Tablet Strengths

\begin{tabular}{|c|c|c|c|c|}
\hline \multirow[b]{2}{*}{$\mathrm{pH}$} & \multirow{2}{*}{$\begin{array}{l}\text { Solubility }^{a} \\
\text { (mg/mL) }\end{array}$} & \multicolumn{3}{|c|}{$D / S^{b}(\mathrm{~mL})$} \\
\hline & & $200 \mathrm{mg}^{c}$ & $400 \mathrm{mg}$ & $800 \mathrm{mg}^{d}$ \\
\hline 1.2 & $>3.5$ & $<57$ & $<114$ & $<229$ \\
\hline 4.5 & $\sim 2.6$ & $\sim 77$ & 154 & $\sim 308^{e}$ \\
\hline 5.8 & $\sim 2.3$ & $\sim 87$ & 174 & $\sim 348^{e}$ \\
\hline 6.8 & $\sim 2.4$ & $\sim 83$ & 167 & $\sim 333^{e}$ \\
\hline 7.4 & $\sim 2.5$ & $\sim 80$ & 160 & $\sim 320^{e}$ \\
\hline \multicolumn{4}{|c|}{$\begin{array}{l}{ }^{a} \text { Data read from the plot published by Shojaei et al. }{ }^{48} \\
{ }^{b} \text { Critical limit: }<250 \mathrm{~mL} .{ }^{2-4} \\
{ }^{c} \text { Strength of WHO Essential Medicines List. }{ }^{19}\end{array}$} & the USA \\
\hline
\end{tabular}




\section{Dosage Form Strengths}

The WHO Model List of Essential Medicines lists a dosage strength of $200 \mathrm{mg}$ for aciclovir. ${ }^{19}$ Most European countries have Marketing Authorizations (MAs) for IR solid oral dosage forms of 200 and $400 \mathrm{mg}$, but some countries also have an MA for a $800 \mathrm{mg}$ strength, see Table 2. The USA has MA's for IR solid oral dosage forms of 200, 400, and $800 \mathrm{mg} .{ }^{52}$

\section{PHARMACOKINETIC PROPERTIES}

\section{Permeability and Absorption}

Data found in the literature for Caco-2 permeability are summarized in Table 3 .

A permeability study employing $3 \mathrm{H}$-aciclovir indicated an apparent permeability coefficient of about $1.19 \times 10^{-5} \mathrm{~cm} / \mathrm{s},{ }^{53}$ but other reports obtained considerably lower values, ranging from of $0.12 \times 10^{-644}$ to $2.0 \times 10^{-6} \mathrm{~cm} / \mathrm{s},{ }^{54}$ see Table 3 . As drugs with a permeability in the range 70$100 \%$ absorbed usually have a $P_{\text {app }}$ value greater than $10 \times 10^{-6} \mathrm{~cm} / \mathrm{s},{ }^{54}$ most of these data suggest that permeability of aciclovir is low. It has also been hypothesized that a $\log P$ value greater than that of metoprolol (1.72) indicates high permeability. ${ }^{51}$ As all $\log P$ values reported for aciclovir lie far below that value (see above), according to this paradigm aciclovir is expected to have low permeability.

Aciclovir's absolute BA following oral administration has been reported to be in a range of 10 $30 \%$ in humans. ${ }^{18,22,23,25}$ This poor systemic BA is considered to be a result of the characteristics of the drug itself and not its delivery vehicle. ${ }^{18,25}$ Its absorption occurs mainly by passive diffusion mechanism and it is slow, variable and incomplete. ${ }^{23,55}$ Maximum plasma concentrations are reached within 1.5-2.5 h. ${ }^{18,22,23,55,56}$ Aciclovir shows a two compartment pharmacokinetic behavior, regardless of the dosage, duration of treatment or frequency of administration. ${ }^{36}$ After multiple dose administration, steady-state concentrations are reached in 1-2 days. ${ }^{18}$ Some studies suggest that increasing doses result in decreasing $\mathrm{BA}^{23,25,35}$ or less than proportional increases in $C_{\max }{ }^{57}$ and it has been suggested that this behavior may be due to a saturable carrier system or a limited area for absorption in the gastro-intestinal (GI) tract or to the low solubility of this API. ${ }^{18,46,55}$ Other studies found near- proportional increases in AUC with increasing doses in the dose range used clinically of 100 $800 \mathrm{mg} .{ }^{34,47}$ Food does not appear to affect the rate and extent of absorption. ${ }^{18,23,25,55}$

\section{Distribution}

Aciclovir is widely distributed into most body tissues, including the brain, kidney, lung, liver, heart tissue, muscle, spleen, placenta, uterus, vaginal mucosa and secretions, semen, saliva, amniotic fluid, aqueous humor and cerebrospinal fluid. ${ }^{18,23}$ Aciclovir demonstrates minimal protein binding (9-33\%) at therapeutic plasma concentrations. ${ }^{18,23,24}$

\section{Metabolism and Excretion}

Most of a single aciclovir dose (62-91\% of an intravenous dose) is excreted unchanged in urine via glomerular filtration and tubular secretion, in adults with normal renal function. ${ }^{23,24}$ Aciclovir is metabolized in the liver, partially to 9-(carboxymethoxy)methyl]guanine (CMMG) and minimally to 8-[(hydroxy-9-(2-hydroxiethoxy)methyl]guanine. ${ }^{18,23,24}$ The only known urinary metabolite is CMMG. Plasma concentrations of aciclovir appear to decline in a biphasic manner. In adults with normal renal function, $t_{1 / 2} \alpha$ averages $0.34 \mathrm{~h}$ and $t_{1 / 2} \beta$ averages $2.1-3.5 \mathrm{~h}^{18,23,24,55}$

\section{DOSAGE FORM PERFORMANCE}

\section{Bioequivalence of Different Formulations}

Six in vivo BE studies comparing drug products with the innovator drug product, Zovirax ${ }^{\circledR}$, were identified. Details of these studies are presented in Table 4. In all of them, the comparator and the generic formulations were bioequivalent. ${ }^{58-62}$

Moreover, in some Summary of Products Characteristics (SmPCs) of drug products with a $\mathrm{MA}$ in DE, results of successful in vivo BE studies are reported. The SmPCs of Aciclovir-CT 200/400/ $800 \mathrm{mg}$ Tabletten ${ }^{63}$ reports in vivo BE studies of each of the three tablet strengths, while the SmPC of Virzin 200/400/800 $\mathrm{mg}^{63}$ reports in vivo BE studies of the 400 and $800 \mathrm{mg}$ strength. All these studies used 20 volunteers and BE was established according to AUC, $C_{\max }$, and $T_{\text {max. }}$ It is very 
Table 2. Excipients* Present in Aciclovir IR Solid Oral Drug Products ${ }^{\dagger}$ with a Marketing Authorization (MA) in Germany (DE), Denmark (DK), Finland (FI), France (FR), The Netherlands (NL), Norway (NO), Spain (ES) and Sweden (SE), ${ }^{\ddagger}$ and the Minimal and Maximal Amount of that Excipient Present Pro Dosage Unit in Solid Oral Drug Products with a MA in the USA ${ }^{s}$

\begin{tabular}{|c|c|c|}
\hline Excipient & $\begin{array}{l}\text { Drug Products Containing that Excipient } \\
\text { with a MA Granted by the Named Country }\end{array}$ & $\begin{array}{l}\text { Range Present in } \\
\text { Solid Oral Dosage Forms } \\
\text { with a MA in the USA (mg) }\end{array}$ \\
\hline $\begin{array}{l}\text { Aluminum } \\
\text { magnesium silicate }{ }^{a}\end{array}$ & $\mathrm{DE}(1,2)$ & $8-24$ \\
\hline Cellulose & $\begin{array}{l}\mathrm{DE}(1-16), \mathrm{DK}(17-23), \mathrm{ES}(24-37), \mathrm{FI}(38-47), \mathrm{FR} \\
\quad(48-64), \mathrm{NL}(65-73), \mathrm{NO}(74-79), \mathrm{SE}(80-87)\end{array}$ & $4.6-1385^{b}$ \\
\hline Crospovidone & $\begin{array}{l}\mathrm{DE}(3,6-14,16), \mathrm{DK}(18-20), \mathrm{ES}(24), \mathrm{FI}(39,41,43) \\
\quad \mathrm{NL}(69), \mathrm{NO}(76), \mathrm{SE}(82,83)\end{array}$ & $86-500$ \\
\hline Croscarmellose & $\operatorname{ES}(27)$ & \\
\hline Gelatin & $\mathrm{SE}(85)$ & $1-756^{b}$ \\
\hline Hypromellose & $\mathrm{DE}(1,2), \mathrm{FI}(44,45), \mathrm{NO}(77), \mathrm{SE}(80)$ & $0.8-86$ \\
\hline Lactose & $\begin{array}{l}\mathrm{DE}(3,4,6,9,12,14), \mathrm{DK}(18,19,21), \mathrm{ES}(25,28,30,32,34,36), \\
\quad \mathrm{FI}(39,41,46), \mathrm{FR}(50,54,58,60,63), \mathrm{NL}(69,71) \\
\quad \mathrm{NO}(76,78), \operatorname{SE}(82,85,86)\end{array}$ & $23-1020^{b}$ \\
\hline Macrogol & $\mathrm{DE}(1,2)$ & $0.12-500^{b}$ \\
\hline Magnesium stearate & $\begin{array}{l}\mathrm{DE}(1-16), \mathrm{DK}(17-23), \mathrm{ES}(24-37), \mathrm{FI}(38-47), \mathrm{FR}(48-64), \\
\quad \mathrm{NL}(65-73), \mathrm{NO}(74-79), \mathrm{SE}(80-84,86,87)\end{array}$ & $0.15-401^{b}$ \\
\hline Povidone & $\begin{array}{l}\mathrm{DE}(1,2,4,5,15), \mathrm{DK}(21-23), \mathrm{ES}(25-37), \mathrm{FI}(38,42,46,47), \\
\quad \mathrm{FR}(48-64), \mathrm{NL}(65-68,70-72), \mathrm{NO}(75,78,79), \mathrm{SE}(84,86,87)\end{array}$ & $0.17-75$ \\
\hline Silica & $\begin{array}{l}\mathrm{DE}(7,8,10,11,13,15,16), \mathrm{DK}(17,20,23) \\
\quad \mathrm{ES}(24,26,29,31,33,35,37), \mathrm{FI}(38,40-43), \mathrm{FR}(48,49,52,53 \\
56,57,61,62), \mathrm{NL}(66-68,70,73), \mathrm{NO}(74,75), \mathrm{SE}(81,83,84)\end{array}$ & $0.65-99$ \\
\hline $\begin{array}{l}\text { Sodium starch } \\
\text { glycolate }\end{array}$ & $\begin{array}{l}\mathrm{DE}(1-16), \mathrm{DK}(17-23), \mathrm{ES}(24-26,28-37), \mathrm{FI}(38-47) \\
\quad \mathrm{FR}(48-64), \mathrm{NL}(65-73), \mathrm{NO}(74-79), \mathrm{SE}(80-87)\end{array}$ & $2-876^{b}$ \\
\hline $\begin{array}{l}\text { Sodium stearyl } \\
\text { fumarate }\end{array}$ & $\mathrm{SE}(85)$ & $1.2-24$ \\
\hline Starch & $\mathrm{DE}(15), \mathrm{SE}(85)$ & $0.44-1135^{b}$ \\
\hline Starch, pregelatinized & DK(17), FI(40), NL(73), NO(74), SE (81) & $6.6-600$ \\
\hline
\end{tabular}

1. Zovirax ${ }^{\circledR} 200 \mathrm{mg} /-400 \mathrm{mg} /-800 \mathrm{mg}$ Filmtabletten (Mono)

2. Acyclovir-ratiopharm ${ }^{\circledR} 200 \mathrm{mg} /-400 \mathrm{mg} /-800$ Filmtabletten (Mono)

3. ACERPES ${ }^{\circledR} 800 \mathrm{mg}$ Tabletten (Mono)

4. Acic $^{\circledR} 200$ Tabletten (Mono)

5. Acic $^{\circledR} 400 \mathrm{mg} /-800 \mathrm{mg}$ Tabletten (Mono)

6. Aciclobeta ${ }^{\circledR} 200$ Tabletten (Mono)

7. Aciclobeta ${ }^{\circledR} 400 \mathrm{mg} /-800 \mathrm{mg}$ Tabletten (Mono)

8. Aciclostad $^{\mathbb{R}} 200 \mathrm{mg} /-400 \mathrm{mg} /-800 \mathrm{mg}$ Tabletten (Mono)

9. Acyclovir $200 \mathrm{mg} /-400 \mathrm{mg}-1 \mathrm{~A}$ Pharma ${ }^{\circledR}$ Tabletten (Mono)

10. Acyclovir 800-1 A Pharma ${ }^{\circledR}$ Tabletten (Mono)

11. Acyclovir AL $200 \mathrm{mg} /-400 \mathrm{mg} /-800 \mathrm{mg}$ Tabletten (Mono)

12. Acyclovir-CT $200 \mathrm{mg}$ Tabletten (Mono)

13. Acyclovir-CT $400 \mathrm{mg} /-800 \mathrm{mg}$ Tabletten (Mono)

14. Herpetad ${ }^{\circledR} 200 \mathrm{mg}$ Tabletten (Mono)

15. Virzin $200 \mathrm{mg} /-400 \mathrm{mg}$ Tabletten (Mono)

16. Virzin 800 Tabletten (Mono)

17. Acyclovir Ranbaxy, tabletter $200 \mathrm{mg} /-400 \mathrm{mg} /-800 \mathrm{mg}$

18. Acyclovir 1A Farma, tabletter $200 \mathrm{mg} /-400 \mathrm{mg} /-800 \mathrm{mg}$

19. Acyclovir HEXAL, tabletter $200 \mathrm{mg} /-400 \mathrm{mg} /-800 \mathrm{mg}$

20. Aciclodan, tabletter $200 \mathrm{mg} /-400 \mathrm{mg} /-800 \mathrm{mg}$ 
Table 2. (Continued)

21. Zovir, tabletter $200 \mathrm{mg}$

22. Zovir, tabletter $400 \mathrm{mg} /-800 \mathrm{mg}$

23. Acyclovir Actavis, tabletter $200 \mathrm{mg} /-400 \mathrm{mg} /-800 \mathrm{mg}$

24. Aciclostad $^{\mathbb{R}} 200 /-800$, comprimidos de $200 \mathrm{mg} /-800 \mathrm{mg}$

25. ACICLOVIR BEXAL $200 \mathrm{mg}$ Comprimidos EFG

26. ACICLOVIR BEXAL $800 \mathrm{mg}$ Comprimidos EFG

27. ACICLOVIR COMBINO PHARM $800 \mathrm{mg}$ EFG

28. ACICLOVIR CUVEFARMA $200 \mathrm{mg}$ comprimidos EFG

29. ACICLOVIR CUVEFARMA $800 \mathrm{mg}$ comprimidos EFG

30. ACICLOVIR KORHISPANA $200 \mathrm{mg}$ comprimidos EFG

31. ACICLOVIR KORHISPANA $800 \mathrm{mg}$ comprimidos EFG

32. ACICLOVIR MERCK $200 \mathrm{mg}$ comprimidos EFG

33. ACICLOVIR MERCK $800 \mathrm{mg}$ comprimidos EFG

34. ACICLOVIR RANBAXY $200 \mathrm{mg}$ COMPRIMIDOS EFG

35. ACICLOVIR RANBAXY $800 \mathrm{mg}$ COMPRIMIDOS EFG

36. ACICLOVIR STADA $200 \mathrm{mg}$ COMPRIMIDOS EFG

37. ACICLOVIR STADA $800 \mathrm{mg}$ COMPRIMIDOS EFG

38. Acyclovir Alpharma $200 \mathrm{mg} /-400 \mathrm{mg} /-800 \mathrm{mg}$ tabletti

39. Acyclovir HEXAL $200 \mathrm{mg} /-400 \mathrm{mg} /-800 \mathrm{mg}$ tabletti

40. Acyclovir Ranbaxy $200 \mathrm{mg} /-400 \mathrm{mg} /-800 \mathrm{mg}$ tabletti

41. Aclovir $200 \mathrm{mg} /-400 \mathrm{mg}$ tabletti

42. Aclovir $800 \mathrm{mg}$ tabletti

43. Acyclostad $200 \mathrm{mg} /-400 \mathrm{mg} /-800 \mathrm{mg}$ tabletti

44. ACYRAX $200 \mathrm{mg} /-400 \mathrm{mg} /-800 \mathrm{mg}$ tabletti

45. Asikloviiri Orion $200 \mathrm{mg} /-400 \mathrm{mg} /-800 \mathrm{mg}$ tabletti

46. Zovirax $200 \mathrm{mg}$ tabletti

47. Zovirax $400 \mathrm{mg} /-800 \mathrm{mg}$ tabletti

48. ACYCLOVIR ALMUS $200 \mathrm{mg} \mathrm{cp}$

49. ACYCLOVIR ARROW $200 \mathrm{mg} /-800 \mathrm{mg} \mathrm{cp}$

50. ACYCLOVIR BIOGARAN $200 \mathrm{mg} \mathrm{cp}$

51. ACYCLOVIR BIOGARAN $800 \mathrm{mg} \mathrm{cp}$

52. ACYCLOVIR EG $200 \mathrm{mg} /-800 \mathrm{mg} \mathrm{cp}$

53. ACYCLOVIR G GAM $200 \mathrm{mg} \mathrm{cp}$

54. ACYCLOVIR MERCK $200 \mathrm{mg} \mathrm{cp}$

55. ACYCLOVIR MERCK $800 \mathrm{mg} \mathrm{cp}$

56. ACYCLOVIR QUALIMED $200 \mathrm{mg} /-800 \mathrm{mg} \mathrm{cp}$

57. ACYCLOVIR RATIOPHARM $200 \mathrm{mg} /-800 \mathrm{mg}$ cp

58. ACYCLOVIR RPG $200 \mathrm{mg} \mathrm{cp}$

59. ACYCLOVIR RPG $800 \mathrm{mg} \mathrm{cp}$

60. ACYCLOVIR SANDOZ $200 \mathrm{mg} \mathrm{cp}$

61. ACYCLOVIR SANDOZ $800 \mathrm{mg} \mathrm{cp}$

62. ACYCLOVIR TEVA $200 \mathrm{mg} /-800 \mathrm{mg} \mathrm{cp}$

63. ZOVIRAX $200 \mathrm{mg} \mathrm{cp}$

64. ZOVIRAX $800 \mathrm{mg} \mathrm{cp}$

65. Acyclovir $200 /-400 /-800$, tabletten $200 /-400 /-800 \mathrm{mg}$

66. Acyclovir CF $200 \mathrm{mg} /-400 \mathrm{mg} /-800 \mathrm{mg}$, tabletten

67. Acyclovir $200 \mathrm{mg} /-400 \mathrm{mg} /-800 \mathrm{mg}$, tabletten

68. Acyclovir ratiopharm $200 \mathrm{mg} /-400 \mathrm{mg} /-800 \mathrm{mg}$, tabletten

69. Acyclovir Sandoz tablet 200/-400/-800, tabletten $200 /-400 /-800 \mathrm{mg}$

70. Acyclovir $200 /-400 /-800 \mathrm{PCH}$, tabletten $200 /-400 /-800 \mathrm{mg}$

71. Acyclovir $200 \mathrm{mg}$, tabletten

72. Acyclovir $400 \mathrm{mg}$, tabletten

73. Acyclovir $200 \mathrm{mg} /-400 \mathrm{mg} /-800 \mathrm{mg}$, tabletten

74. Acyclovir tabletter Ranbaxy $200 \mathrm{mg} /-400 \mathrm{mg} /-800 \mathrm{mg}$

75. Acyclovir Alpharma $200 \mathrm{mg} /-400 \mathrm{mg} /-800 \mathrm{mg}$ tabletter 
Table 2. (Continued)

76. Acyclovir HEXAL $200 \mathrm{mg} /-400 \mathrm{mg} /-800 \mathrm{mg}$ tabletter

77. Aciklovir Merck NM $200 \mathrm{mg} /-400 \mathrm{mg} /-800 \mathrm{mg}$ tabletter

78. Zovirax tabletter $200 \mathrm{mg}$

79. Zovirax tabletter $400 \mathrm{mg} /-800 \mathrm{mg}$

80. Acyclovir Merck NM $200 \mathrm{mg} /-400 \mathrm{mg} /-800 \mathrm{mg}$ tabletter

81. Acyclovir Ranbaxy $200 \mathrm{mg} /-400 \mathrm{mg} /-800 \mathrm{mg}$

82. Acyclovir Sandoz $200 \mathrm{mg} /-400 \mathrm{mg} /-800 \mathrm{mg}$, tabletter

83. Aciklovir STADA $200 \mathrm{mg} /-400 \mathrm{mg} /-800 \mathrm{mg}$ tabletter

84. Acyclovir Alpharma $200 \mathrm{mg} /-400 \mathrm{mg} /-800 \mathrm{mg}$ tabletter

85. Geavir $200 \mathrm{mg} /-400 \mathrm{mg} /-800 \mathrm{mg}$ tabletter

86. Zovirax $200 \mathrm{mg}$ tabletter

87. Zovirax $400 \mathrm{mg} /-800 \mathrm{mg}$ tabletter

${ }^{*}$ Colourants and water are not included.

${ }^{\dagger}$ Excluded are: dispersible tablets, oral suspension, and oral solutions.

${ }^{\ddagger}$ DE: www.rote-liste.de (assessed February 28, 2007); DK: www.dkma.dk (assessed February 28, 2007); FI: www.nam.fi (assessed February 28, 2007); FR: www.vidal.fr (assessed February 28, 2007); NL: www.cbg-meb.nl. (assessed February 27, 2007); NO: www.legemiddelverket.no (assessed March 1, 2007); ES: www.agemed.es (assessed February 28, 2007); SE: www. lakemedelsverket.se (assessed March 1, 2007).

${ }^{\S}$ USA: http://www.fda.gov/cder/iig/iigfaqweb.htm\#purpose (version date January 9, 2007).

${ }^{a}$ Includes aluminum magnesium silicate and aluminum magnesium trisilicate.

${ }^{b}$ The upper range value reported is unusual high for solid oral dosage forms and the authors doubt on its correctness.

\section{Excipients}

In the above-mentioned $\mathrm{BE}$ studies, various excipients were used in the formulations. Aciclovir-CT tabletten contain cellulose, crospovidone, magensium stearate, sodium starch glycolate, lactose (200 and $400 \mathrm{mg}$ strengths only) and silica (800 mg strength only); ${ }^{63}$ Virzin contains cellulose; magnesium stearate silica, sodium starch glycolate, starch (200 and $400 \mathrm{mg}$ strengths only), crospovidone (800 mg strength only) and povidone (200 and $400 \mathrm{mg}$ strengths only), ${ }^{63}$ whereas Zovirax ${ }^{\circledR}$ contains aluminum magnesium silicate, cellulose, hypromellose, macrogol, magnesium stearate, povidone, and sodium starch glycolate; ${ }^{63}$ see also Table 2.

A study carried out with Caco-2 showed that, of nine common excipients studied, only sodium lauryl sulfate slightly increased the permeability of aciclovir, whereas lactose, hydroxypropyl methyl cellulose, Tween 80, propylene glycol, polyethylene glycol 400, dioctyl sodium sulfosuccinate, sodium EDTA and anhydrous cherry flavor, had no effect on the permeability. ${ }^{64}$

Excipients present in aciclovir IR solid oral drug products with a MA in Germany (DE), Denmark (DK), Finland (FI), France (FR), The Netherlands (NL), Norway (NO), Spain (ES) and Sweden (SE), are shown in Table 2. Additionally, the minimum and maximum amount of the listed excipients present per dosage unit in solid oral drug products with a MA in the USA, taken from the FDA Inactive Ingredient Data Base ${ }^{65}$ is indicated.

It can be expected that the drug products having a MA in the reported countries successfully passed an in vivo BE study. In $\mathrm{DE}$, the exemption from demonstration of $\mathrm{BE}$ in vivo that existed for some APIs in the 1980s and 1990s was not applied to

Table 3. Apparent Permeability of Aciclovir in Caco-2 Cell Monolayers

\begin{tabular}{lccl}
\hline $\begin{array}{l}\text { Permeability } \\
\left(\times 10^{-6} \mathrm{~cm} / \mathrm{s}\right)\end{array}$ & $\mathrm{pH}$ & Initial Concentration & \multicolumn{1}{c}{ References } \\
\hline 0.12 & 7.4 & $0.02-6 \mathrm{mM}$ (not specified) & Bergstrom et al..$^{44}$ \\
0.25 & 7.4 & $0.01-0.1 \mathrm{mM}$ (not specified) & Yazdanian et al. ${ }^{74}$ \\
0.43 & 7.4 & Not specified & Friedrichsen et al. $^{75}$ \\
1.08 & - & Not specified & de Vrueh et al. ${ }^{76}$ \\
1.26 & 6.8 & Below saturation & Rege et al. $^{64}$ \\
2.00 & $6.5(\mathrm{~A}), 7.4(\mathrm{~B})$ & Not specified & Yee \\
11.9 & 7 & $0.5 \mathrm{mCi} / \mathrm{mL}$ (radioactive ACV) & Salama et al. $^{50}$ \\
\hline
\end{tabular}

(A), apical compartment; (B), basolateral compartment. 
Table 4. Aciclovir Bioequivalence Studies Found in Literature

\begin{tabular}{|c|c|c|c|c|c|}
\hline Reference & $\begin{array}{l}\text { Composition of } \\
\text { Test Formulation }\end{array}$ & Study Design & $\begin{array}{l}\text { Number } \\
\text { of Subjects }\end{array}$ & $\begin{array}{c}\text { Estimates } \\
\text { for BE }\end{array}$ & Result \\
\hline Al-Yamani et al. ${ }^{61}$ & $400 \mathrm{mg}$ tablet & 2 -way crossover & 24 & $\begin{array}{c}C_{\max } \\
\mathrm{AUC}_{0-t} \\
\mathrm{AUC}_{0-\infty} \\
C_{\max } / \mathrm{AUC}_{0-\infty} \\
T_{\max }\end{array}$ & $\begin{array}{c}\text { Test formulation } \\
\text { bioequivalent }\end{array}$ \\
\hline Bahrami et al. ${ }^{62}$ & $400 \mathrm{mg}$ tablet & Non crossover & 12 & $\begin{array}{c}T_{\max } \\
C_{\max } \\
\mathrm{AUC}_{0-72} \\
\mathrm{AUC}_{0-\infty} \\
T_{1 / 2}\end{array}$ & $\begin{array}{c}\text { Test formulation } \\
\text { bioequivalent }\end{array}$ \\
\hline $\begin{array}{l}\text { Rojanasthien } \\
\text { et al. }{ }^{60}\end{array}$ & $200 \mathrm{mg}$ tablet & $\begin{array}{r}\text { Two-period } \\
\text { crossover }\end{array}$ & Not reported & $\begin{array}{c}T_{1 / 2} \\
T_{\max } \\
\mathrm{AUC}_{0-\infty} \\
C_{\max }\end{array}$ & $\begin{array}{c}\text { Test formulation } \\
\text { bioequivalent }\end{array}$ \\
\hline $\begin{array}{l}\text { Vergin et al. }{ }^{58} ; \\
1 \text { st study }\end{array}$ & $\begin{array}{c}200 \mathrm{mg} \text { tablet }+ \\
250 \mathrm{mg} \\
\text { IV infusion }\end{array}$ & Three-way & 24 & $\begin{array}{c}C_{\max } \\
T_{\max } \\
\mathrm{AUC}_{0-t} \\
\text { Dose renally } \\
\text { excreted }\end{array}$ & $\begin{array}{c}\text { Test formulation } \\
\text { bioequivalent }\end{array}$ \\
\hline $\begin{array}{l}\text { Vergin et al. }{ }^{58} ; 2 \text { nd } \\
\text { study }\end{array}$ & $400 \mathrm{mg}$ tablet & Two-way & 24 & $\begin{array}{c}C_{\max } \\
T_{\max } \\
\mathrm{AUC}_{0-t} \\
\text { Dose renally } \\
\text { excreted }\end{array}$ & $\begin{array}{c}\text { Test formulation } \\
\text { bioequivalent }\end{array}$ \\
\hline Yuen et al. ${ }^{59}$ & Not reported & Two-way crossover & 12 & $\begin{array}{c}\mathrm{AUC}_{0-\infty} \\
C_{\max } \\
T_{\max } \\
K_{\mathrm{el}}, t_{1 / 2}, V_{\mathrm{d}}\end{array}$ & $\begin{array}{c}\text { Test formulation } \\
\text { bioequivalent }\end{array}$ \\
\hline
\end{tabular}

aciclovir. ${ }^{66}$ Similarly, aciclovir is not on the list of APIs exempted from demonstration of $\mathrm{BE}$ in vivo for IR solid oral drug products seeking a national MA only in The Netherlands. ${ }^{67}$

\section{Dissolution}

The USP 29 dissolution specifications for Aciclovir Tablets are not less than $80 \%$ (Q) of the labeled amount dissolved within $45 \mathrm{~min}$ in $900 \mathrm{~mL}$ of $0.1 \mathrm{~N}$ hydrochloric acid, using the paddle at $50 \mathrm{rpm}$, and for Aciclovir Capsules the specification is not less than $75 \%$ (Q) within $45 \mathrm{~min}$ in $900 \mathrm{~mL}$ of $0.1 \mathrm{~N}$ hydrochloric acid, using the basket at $100 \mathrm{rpm}$, respectively. ${ }^{20}$ The dissolution of the innovator and five generic drug products, each containing $800 \mathrm{mg}$ aciclovir, was compared using USP apparatus 2 and USP apparatus 3. The dissolution of all drug products was complete within in 15 min, in both apparatus. ${ }^{68}$

One of the six in vivo $\mathrm{BE}$ studies mentioned above compared the dissolution of innovator and generic drug products as stipulated by USP XXIII, finding them to be not statistically different. ${ }^{61}$

\section{DISCUSSION}

\section{Solubility}

$\mathrm{D} / \mathrm{S}$ values for different $\mathrm{pHs}$ are shown in Table 1 . The WHO recommended dosage strength and the $400 \mathrm{mg}$ strength qualify as highly soluble according to all Guidelines. ${ }^{2-4}$ However, in some markets, the highest dosage strength is $800 \mathrm{mg}$, for which that criterion is not met.

\section{Permeability}

The BA for aciclovir ranges from $10 \%$ to $30 \%$, indicating, given the small amount of metabolism, that this API is not highly permeable according to all Guidelines. ${ }^{2-4}$ Caco-2 permeability data and $\log P_{\text {oct }}$ values support that conclusion. 


\section{BCS Classification}

Combining the data on solubility and permeability, according to all Guidelines, ${ }^{2-4}$ the 200 and $400 \mathrm{mg}$ tablet strengths are BCS Class III. Kasim et al. ${ }^{51}$ also classified aciclovir as BCS Class III, but their classification was based on correlations of partition coefficients with permeability, a method not supported by HHS-FDA and other regulatory authorities due to its limited predictability. Lindenberg et al. ${ }^{69}$ and the recently adopted revised WHO Guideline ${ }^{2}$ also classify aciclovir as BCS Class III. Wu and Benet, ${ }^{70}$ using the disposition characteristics of the API as estimate for its permeability, assigned aciclovir to Class III of their Biopharmaceutics Drug Disposition Classification System (BDDCS). For $\mathrm{pH}$ values 4.5 and above, the $800 \mathrm{mg}$ tablet strength shows D/S ratio's outside the $250 \mathrm{~mL}$ limit. If $800 \mathrm{mg}$ is taken as highest tablet strength, aciclovir would be classified in BCS Class IV.

\section{Surrogate Techniques for In Vivo Bioequivalence Testing}

The low permeability of aciclovir is the limiting step in its absorption process. Bioinequivalence caused by differences in GI permeability between test and comparator, caused by an excipient effect, can only be circumvented by formulating the test product only with excipients known not to affect GI permeability. Bioinequivalence caused by differences in in vivo disintegration and/or in vivo dissolution between test and comparator, being a potential cause of bioinequivalence in particular for $800 \mathrm{mg}$ tablet strengths, can be excluded by requiring the comparator and the test product to be both very rapidly dissolving, that is, dissolve $85 \%$ in $15 \mathrm{~min}$ or less, using a basket apparatus at $100 \mathrm{rpm}$ or paddle apparatus at $50 \mathrm{rpm}$, in a volume of $900 \mathrm{~mL}$ or less, in the three BCS-media. ${ }^{2,71-73}$

\section{Risks of Excipient and/or Manufacturing to Cause Bioinequivalence}

One report was identified of an excipient interaction, being an in vitro study only, using Caco-2, with sodium laurylsulfate, and the effect was limited. ${ }^{64}$ For the excipients shown in Table 2, it can be supposed that they show no influence on the absorption of aciclovir when present in usual amounts; note that Table 2 does not include sodium laurylsulfate. From a more general perspective, aciclovir seems not to be a critical API with respect to a risk for bioinequivalence: not one single example of bioinequivalence was identified, whereas eight in vivo $\mathrm{BE}$ studies were identified demonstrating in vivo $\mathrm{BE}$ of different formulations. Furthermore, 87 drug products (with MAs) were identified, for which it can be assumed that an in vivo BE study was successfully passed, which contain a wide variety of compositions with respect to excipients.

\section{Patient's Risks Associated with Bioinequivalence}

Aciclovir has a broad therapeutic index. Oral administration of aciclovir is usually well tolerated, and oral overdosing does not provoke serious adverse effects. Oral aciclovir has no lifethreatening indication, and its therapeutic range seems wide enough to open the possibility for a biowaiver.

\section{CONCLUSION}

Aciclovir cannot unequivocally be classified to the BCS because different countries have different highest tablet strengths on their markets, which would lead to different BCS classifications in different countries. But the BCS classification of an API is not a purpose in itself, the relevant question being: is a biowaiver scientifically justified and if justified, which restrictions have to be formulated?

Up to now, the $\mathrm{FDA}^{3}$ and the $\mathrm{EMEA}^{4}$ do not accept biowaivers for BCS Class III and BCS Class IV APIs, so acyclovir would obviously be excluded from consideration for a biowaiver in these jurisdictions at the present time. However, the recently revised WHO guidance ${ }^{2}$ extended the possibility of a biowaiver approval to BCS Class III APIs under certain conditions.

The risk of an acyclovir drug product being bioinequivalent appears to be very low, provided it contains only excipients present in IR aciclovir solid oral drug products approved in ICH or associated countries, for instance those presented in Table 2 of this article, and meets the biowaiver dissolution criteria. Also, the patient risks associated with a bioinequivalence inadvertently not detected by application of in vitro BE test can be deemed acceptable. 
The preponderance of evidence indicates that a biowaiver for IR aciclovir solid oral drug products with strengths up to $800 \mathrm{mg}$ is justified, if: (a) the test product contains only excipients present in aciclovir solid oral drug products approved in ICH or associated countries, for instance as presented in Table 2 of this article, and (b) the comparator and the test product both are very rapidly dissolving, that is, dissolve $85 \%$ in $15 \mathrm{~min}$ or less, using a basket apparatus at $100 \mathrm{rpm}$ or paddle apparatus at $50 \mathrm{rpm}$, in a volume of $900 \mathrm{~mL}$ or less, in pH 1.2, pH 4.5, and pH 6.8 buffers.

\section{ACKNOWLEDGMENTS}

Kik Groot, RIVM, is acknowledged for producing Table 2.

\section{REFERENCES}

1. Vogelpoel H, Welink J, Amidon GL, Junginger HE, Midha KK, Moller H, Olling M, Shah VP, Barends DM. 2004. Biowaiver monographs for immediate release solid oral dosage forms based on biopharmaceutics classification system (BCS) literature data: Verapamil hydrochloride, propranolol hydrochloride, and atenolol. J Pharm Sci 93:1945-1956.

2. WHO. 2006. Proposal to waive in vivo bioequivalence requirements for WHO Model List of Essential Medicines immediate-release, solid oral dosage forms. Technical Report Series, No937, 40th Report, Annex 8 of WHO Expert committee on specifications for pharmaceutical preparations. Available from URL http://whqlibdoc.who.int/trs/WHO_TRS_ 937 eng.pdf.

3. FDA. 2000. Waiver of In Vivo Bioavailability and Bioequivalence Studies for Immediate-Release Solid Oral Dosage Forms Based on a Biopharmaceutics Classification System. Available from URL http://www.fda.gov/cder/guidance/3618fnl.pdf. ed.: U.S. Department of Health and Human Services Food and Drug Administration Center for Drug Evaluation and Research (CDER).

4. EMEA. 2001. Note for guidance on the investigation of bioavailability and bioequivalence. Available from URL http://www.emea.eu.int/pdfs/human/ ewp/140 198en.pdf. ed.: Committee for Proprietary Medicinal Products (CPMP).

5. Kalantzi L, Reppas C, Dressman JB, Amidon GL, Junginger HE, Midha KK, Shah VP, Stavchansky SA, Barends DM. 2006. Biowaiver monographs for immediate release solid oral dosage forms: Acetaminophen (paracetamol). J Pharm Sci 95:414.
6. Granero GE, Longhi MR, Becker C, Junginger HE, Kopp S, Midha KK, Shah VP, Stavchansky S, Dressman JB, Barends DM. Biowaiver monographs for immediate release solid oral dosage forms: Acetazolamide. J Pharm Sci (in press).

7. Manzo RH, Olivera ME, Amidon GL, Shah VP, Dressman JB, Barends DM. 2006. Biowaiver monographs for immediate release solid oral dosage forms: Amitriptyline hydrochloride. J Pharm Sci 95:966-973.

8. Verbeeck RK, Junginger HE, Midha KK, Shah VP, Barends DM. 2005. Biowaiver monographs for immediate release solid oral dosage forms based on biopharmaceutics classification system (BCS) literature data: Chloroquine phosphate, chloroquine sulfate, and chloroquine hydrochloride. J Pharm Sci 94:1389-1395.

9. Jantratid E, Prakongpan S, Dressman JB, Amidon GL, Junginger HE, Midha KK, Barends DM. 2006. Biowaiver monographs for immediate release solid oral dosage forms: Cimetidine. J Pharm Sci 95:974984.

10. Becker C, Dressman JB, Amidon GL, Junginger HE, Kopp S, Midha KK, Shah VP, Stavchansky S, Barends DM. 2008. Biowaiver, monographs for immediate release solid oral dosage forms: Ethambutol dihydrochloride. J Pharm Sci 97:1350-1360.

11. Potthast H, Dressman JB, Junginger HE, Midha KK, Oeser H, Shah VP, Vogelpoel H, Barends DM. 2005. Biowaiver monographs for immediate release solid oral dosage forms: Ibuprofen. J Pharm Sci 94:2121-2131.

12. Becker C, Dressman JB, Amidon GL, Junginger HE, Kopp S, Midha KK, Shah VP, Stavchansky S, Barends DM. 2007. Biowaiver monographs for immediate release solid oral dosage forms: Isoniazid. J Pharm Sci 96:522-531.

13. Vogt M, Derendorf H, Kramer J, Junginger HE, Midha KK, Shah VP, Stavchansky S, Dressman JB, Barends DM. 2007. Biowaiver monographs for immediate release solid oral dosage forms: Prednisolone. J Pharm Sci 96:27-37.

14. Vogt M, Derendorf H, Kramer J, Junginger HE, Midha KK, Shah VP, Stavchansky S, Dressman JB, Barends DM. 2007. Biowaiver monographs for immediate release solid oral dosage forms: Prednisone. J Pharm Sci 96:1480-1489.

15. Becker C, Dressman JB, Junginger HE, Kopp S, Shah VP, Stavchansky S, Barends DM. Biowaiver, monographs for immediate release solid oral dosage forms: Pyrazinamide. J Pharm Sci (in press).

16. Kortejarvi H, Yliperttula M, Dressman JB, Junginger HE, Midha KK, Shah VP, Barends DM. 2005. Biowaiver monographs for immediate release solid oral dosage forms: Ranitidine hydrochloride. J Pharm Sci 94:1617-1625.

17. National Library of Medicine (NLM) and the National Institutes of Health, NCBI, 2006. 
PubMed. URL http://www.ncbi.nlm.nih.gov/entrez/ query.fcgi?otool=inlrivmlib.

18. Thomson Healthcare. Micromedex Healthcare Series. http://www.micromedex.com/products/hcs/.

19. World Health Organization, 2005. World Health Organization Model List of Essential Medicines. 15th ed. http://www.who.int/medicines/publications/ EssMedList15.pdf.

20. USP 27 - NF 22. 2006. The United States Pharmacopeia - The National Formulary. ed. The United States Pharmacopeial Convention Inc. Rockville MD: 2085.

21. European Pharmacopoeia 5th edition. ed. Strasbourg, France: Council of Europe, European Directorate for the Quality of Medicines.

22. Consejo General de Colegios Oficiales de Farmacéuticos 2005. Catálogo de Especialidades Farmacéuticas. pp 1316-1317.

23. AHFS Drug Information. 2004. American Society of Hospital Pharmacists (AHFS). Bethesda, MD. pp 765-775.

24. GlaxoSmithKline. 2003. Zovirax for injection. Product Information.

25. GlaxoSmithKline, 2005. Zovirax Prescribing Information.

26. WHOCC. 2006. WHO Collaborating Centre for Drug Statistics Methodology ATC index. ed.

27. O’Brien JJ, Campoli-Richards DM. 1989. Aciclovir: An updated review or its antiviral activity, pharmacokinetic properties and therapeutic efficacy. Drugs 37:233-309.

28. Ellenhorn MJ. 1997. Medical toxicology. Diagnosis and treatment of human poisoning. 2nd subedition. Baltimore: Williams \& Wilkins. pp 296300.

29. Davenport A, Goel S, Mackenzie JC. 1992. Neurotoxicity of aciclovir in patients with end-stage renal failure treated with continuous ambulatory peritoneal dialysis. Am J Kidney Dis 20:647-649.

30. Da Conceicao M, Genco G, Favier JC, Verrot D, Pitti R. 1999. Toxicite cerebrale et renale de l'aciclovir chez un patient traite pour meningo-encephalite [Cerebral and renal toxicity of acyclovir in a patient treated for eningoencephalitis]. Ann Fr Anesth Reanim 18:996-999.

31. Eksborg S, Pal N, Kalin M, Palm C, Soderhall S. 2002. Pharmacokinetics of aciclovir in immunocompromized children with leukopenia and mucositis after chemotherapy: Can intravenous aciclovir be substituted by oral valaciclovir? Med Pediatr Oncol 38:240-246.

32. Landowski CP, Sun D, Foster DR, Menon SS, Barnett JL, Welage LS, Ramachandran C, Amidon GL. 2003. Gene expression in the human intestine and correlation with oral valaciclovir pharmacokinetic parameters. J Pharmacol Exp Ther 306:778-786.

33. Lycke J, Malmestrom C, Stahle L. 2003. Aciclovir levels in serum and cerebrospinal fluid after oral administration of valaciclovir. Antimicrob Agents Chemother 47:2438-2441.

34. MacDougall C, Guglielmo BJ. 2004. Pharmacokinetics of valaciclovir. J Antimicrob Chemother 53: 899-901.

35. Soul-Lawton J, Seaber E, On N, Wootton R, Rolan P, Posner J. 1995. Absolute bioavailability and metabolic disposition of valaciclovir, the L-valyl ester of aciclovir, following oral administration to humans. Antimicrob Agents Chemother 39:27592764.

36. Steingrimsdottir H, Gruber A, Palm C, Grimfors G, Kalin M, Eksborg S. 2000. Bioavailability of aciclovir after oral administration of aciclovir and its prodrug valaciclovir to patients with leukopenia after chemotherapy. Antimicrob Agents Chemother 44:207-209.

37. Anand BS, Katragadda S, Mitra AK. 2004. Pharmacokinetics of novel dipeptide ester prodrugs of aciclovir after oral administration: Intestinal absorption and liver metabolism. J Pharmacol Exp Ther 311:659-667.

38. Thomsen AE, Christensen MS, Bagger MA, Steffansen B. 2004. Aciclovir prodrug for the intestinal di/tri-peptide transporter PEP T1:comparison of in vivo bioavailability in rats and transport in Caco-2 cells. Eur J Pharm Sci 23:319-325.

39. Tolle-Sander S, Lentz KA, Maeda DY, Coop A, Polli JE. 2004. Increased aciclovir oral bioavailability via a bile acid conjugate. Mol Pharm 1:40-48.

40. Hostetler KY, Parker S, Sridhar CN, Martin MJ, Li JL, Stuhmiller LM, van Wijk GM, van den Bosch H, Gardner MF, Aldern KA, Richman DD. 1993. Aciclovir diphosphate dimyristoylglycerol: A phospholipid prodrug with activity against aciclovirresistant herpes simplex virus. Proc Natl Acad Sci USA 90:11835-11839.

41. Kristl A, Srcic S, Vrecer F, Sustar B, Vojnovic D. 1996. Polymorphism and pseudopolymorphism: Influencing the dissolution properties of the guanine derivative aciclovir. Int J Pharm 139:231-235.

42. British Pharmacopoeia. 2007. The Stationery Office on behalf of the Medicines and Healthcare products Regulatory Agency (MHRA).

43. Bergstrom CA, Norinder U, Luthman K, Artursson P. 2002. Experimental and computational screening models for prediction of aqueous drug solubility. Pharm Res 19:182-188.

44. Bergstrom CA, Strafford M, Lazorova L, Avdeef A, Luthman K, Artursson P. 2003. Absorption classification of oral drugs based on molecular surface properties. J Med Chem 46:558-570.

45. Kristl A. 1999. Estimation of aqueous solubility for some guanine derivatives using partition coefficient and melting temperature. J Pharm Sci 88:109110 .

46. Luengo J, Aranguiz T, Sepulveda J, Hernandez L, Von Plessing C. 2002. Preliminary pharmacokinetic 
study of different preparations of aciclovir with beta-cyclodextrin. J Pharm Sci 91:2593-2598.

47. Von Plessing Rossel C, Sepulveda Carreno J, Rodriguez-Baeza M, Bernabe Alderete J. 2000. Inclusion complex of the antiviral drug aciclovir with cyclodextrin in aqueous solution and in solid phase. Quim Nova 23:749-752.

48. Shojaei AH, Berner B, Xiaoling L. 1998. Transbuccal delivery of acyclovir. I. In vitro determination of routes of buccal transport. Pharm Res 15:1182-1188.

49. Kristl A, Pecar S, Kmetec V. 1999. Are calculated $\log \mathrm{P}$ values for some guanine derivatives by different computer programs reliable? Int J Pharm 181:219-226.

50. Balon K, Riebesehl BU, Muller BW. 1999. Drug liposome partitioning as a tool for the prediction of human passive intestinal absorption. Pharm Res 16:882-888.

51. Kasim NA, Whitehouse M, Ramachandran C, Bermejo M, Lennernas H, Hussain AS, Junginger HE, Stavchansky SA, Midha KK, Shah VP, Amidon GL. 2004. Molecular properties of WHO essential drugs and provisional biopharmaceutical classification. Mol Pharm 1:85-96.

52. FDA., 2007. Drugs@FDA: FDA approved drug products.

53. Salama NN, Scott KR, Eddington ND. 2004. DM27, an enaminone, modifies the in vitro transport of antiviral therapeutic agents. Biopharm Drug Dispos 25:227-236.

54. Yee S. 1997. In vitro permeability across Caco-2 cells (colonic) can predict in vivo (small intestinal) absorption in man-fact or myth. Pharm Res 14:763766.

55. Alpha-Pharmaceuticals. 1999. Alpha Aciclovir data sheet.

56. Najib NM, Idkaidek N, Beshtawi M, Mohammed B, Admour I, Alam SM, Dham R. 2005. Bioequivalence assessment of Lovrak (Julphar, UAE) compared with Zovirax (Glaxo Wellcome, UK) - Two brands of Aciclovir-In healthy human volunteers. Biopharm Drug Dispos 26:7-12.

57. University of Texas Libraries. Clinical Pharmacology database. http://www.clinicalpharmacologyip.com.ezproxy.lib.utexas.edu/Forms/Monograph/ monograph.aspx?cpnumU8\&secUmonphar, revision $9 / 28 / 2005$.

58. Vergin H, Kikuta C, Mascher H, Metz R. 1995. Pharmacokinetics and bioavailability of different formulations of aciclovir. Arzneimittelforschung 45:508-515.

59. Yuen KH, Peh KK, Billa N, Chan KL, Toh WT. 1998. Bioavailability and pharmacokinetics of aciclovir tablet preparation. Drug Dev Ind Pharm 24:193-196.

60. Rojanasthien N, Teekachunhatean S, Kumsorn B, Chaichana N, Hay YK. 2002. Bioequivalence study of generic aciclovir compared with the brand name aciclovir. J Med Assoc Thai 85:11211129.

61. Al-Yamani MJ, Al-Khamis KI, El-Sayed YM, Bawazir SA, Al-Rashood KA, Gouda MW. 1998. Comparative bioavailability of two tablet formulations of aciclovir in healthy volunteers. Int J Clin Pharmacol Ther 36:222-226.

62. Bahrami G, Mirzaeei S, Kiani A. 2005. Determination of aciclovir in human serum by high-performance liquid chromatography using liquid-liquid extraction and its application in pharmacokinetic studies. J Chromatogr B Analyt Technol Biomed Life Sci 816:327-331.

63. FachInfo-Service, 2007. Arzneimittel-Informationen für Ärzte und Apotheker. Available at URL www.fachinfo.de.

64. Rege BD, Yu LX, Hussain AS, Polli JE. 2001. Effect of common excipients on Caco-2 transport of low-permeability drugs. J Pharm Sci 90:17761786.

65. FDA, 2007. Inactive Ingredients Database. Available at URL http://www.fda.gov/cder/iig/iigfaqWEB.htm.

66. Gleiter $\mathrm{CH}$, Klotz U, Kuhlmann J, Blume H, Stanislaus F, Harder S, Paulus H, Poethko-Muller C, Holz-Slomczyk M. 1998. When are bioavailability studies required? A German proposal. J Clin Pharmacol 38:904-911.

67. College ter Beoordeling van Geneesmiddelen. 2007. Lijst vrijstelling bio-equivalentieonderzoek (positieve lijst). Available at URL http://www.cbg-meb. $\mathrm{nl} / \mathrm{nl} /$ reghoudr/vrij.htm.

68. Yu LX, Wang JT, Hussain AS. 2002. Evaluation of USP apparatus 3 for dissolution testing of immediate-release products. AAPS PharmSci 4:E1.

69. Lindenberg M, Kopp S, Dressman JB. 2004. Classification of orally administered drugs on the World Health Organization Model list of Essential Medicines according to the biopharmaceutics classification system. Eur J Pharm Biopharm 58:265278.

70. Wu CY, Benet LZ. 2005. Predicting drug disposition via application of BCS: Transport/absorption/ elimination interplay and development of a biopharmaceutics drug disposition classification system. Pharm Res 22:11-23.

71. Polli JE, Yu LX, Cook JA, Amidon GL, Borchardt RT, Burnside BA, Burton PS, Chen ML, Conner DP, Faustino PJ, Hawi AA, Hussain AS, Joshi HN, Kwei G, Lee VH, Lesko LJ, Lipper RA, Loper AE, Nerurkar SG, Polli JW, Sanvordeker DR, Taneja R, Uppoor RS, Vattikonda CS, Wilding I, Zhang G. 2004. Summary workshop report: Biopharmaceutics classification system-Implementation challenges and extension opportunities. J Pharm Sci 93:1375-1381. 
72. Blume HH, Schug BS. 1999. The biopharmaceutics classification system (BCS): Class III drugs-Better candidates for BA/BE waiver? Eur J Pharm Sci 9:117-121.

73. Yu LX, Amidon GL, Polli JE, Zhao H, Mehta MU, Conner DP, Shah VP, Lesko LJ, Chen ML, Lee VH, Hussain AS. 2002. Biopharmaceutics classification system: The scientific basis for biowaiver extensions. Pharm Res 19:921-925.

74. Yazdanian M, Glynn SL, Wright JL, Hawi A. 1998. Correlating partitioning and caco-2 cell permeabil- ity of structurally diverse small molecular weight compounds. Pharm Res 15:1490-1494.

75. Friedrichsen GM, Chen W, Begtrup M, Lee CP, Smith PL, Borchardt RT. 2002. Synthesis of analogs of L-valaciclovir and determination of their substrate activity for the oligopeptide transporter in Caco-2 cells. Eur J Pharm Sci 16:1-13.

76. de Vrueh RL, Smith PL, Lee CP. 1998. Transport of L-valine-aciclovir via the oligopeptide transporter in the human intestinal cell line, Caco-2. J Pharmacol Exp Ther 286:1166-1170. 